\title{
Apoptosis in Trophoblast in Pre-eclampsia and Intrauterine Growth Retardation: A Light Microscopy Study
}

\author{
Pandey Swati, Hemalatha.A*, Malingere Lingaih Harendra Kumar and Munikrishna.M \\ Department of Pathology, Sri Devraj Urs Medical College, Kolar, India
}

\begin{abstract}
Background: Pre-eclampsia and Intrauterine growth retardation (IUGR) affects 3\% and 10\% of all pregnancies. Apoptosis, a programmed cell death is known to occur as a part of both physiological and pathological process. This study was done to calculate apoptotic index in placental trophoblasts of pre-eclampsia, IUGR and compare it with normal term placenta. The aim of this study is to calculate apoptotic index in cytotrophoblast and syncytiotrophoblast in preeclampsia and IUGR and compare it with placenta from normal pregnancy

Methods: A total of fifty four placentas were included in the study. 18/54 cases were from pre-eclampsia patients, 18/54 cases were from IUGR patients, 18/54 were age matched controls. Hematoxylin and eosin stained sections from representative were screened under oil immersion using Olympus microscope (100X) for apoptotic bodies and apoptotic index was calculated by counting the total number of apoptotic bodies and dividing it by total number of nuclei counted in the trophoblasts multipled by 100. Data was entered into Microsoft excel data sheet and was analyzed using SPSS 22 version software. ANOVA was the test of significance used. $\mathrm{P}$ value $<0.05$ was considered significant.
\end{abstract}

Result: Apoptotic bodies and index were maximum in cytotrophoblast and syncytiocytotrophoblast of preeclampsia as compared with IUGR and normal placentas. Statistically significant difference was observed in apoptotic index in cytotrophoblast and apoptotic index in Syncytiotrophoblast between three groups.

Conclusion: Increased number of apoptotic bodies and index were seen in trophoblast of pre-eclampsia cases as compared with IUGR and normal placentas which may be related to onset of maternal inflammatory syndrome.

Keywords: Preeclampsia, Intrauterine Growth Retardation, Apoptosis, Trophoblasts

\section{Introduction}

Preeclampsia and Intrauterine growth retardation (IUGR) affects approximately $3 \%$ and $10 \%$ of all pregnancies worldwide. ${ }^{[1,2]}$ Pathogenesis of both these conditions are linked to placental dysfunction. However the exact cause of placental pathology in both of these conditions are unknown. Apoptosis, a programmed cell death is crucial to the development and homeostasis of human tissue including human placenta. ${ }^{[3]}$ The cause and pathological implication of this phenomenon in preeclampsia and IUGR is unknown.Numerous theories have been proposed to explain the pathogenesis of preeclampsia. One study proposed that major defect in preeclampsia is inadequate remodeling of spiral arteries by trophoblast which reduces the blood supply to the placenta. ${ }^{[4]}$ while another study proposed that prolonged hypoxia in the intervillous space causes oxidative stress and placental apoptosis in conditions like pre-eclampsia and IUGR.$^{[5]}$

Newer pathogenesis proposed that there is release of syncytiotrophoblastic microparticles from apoptotic trophoblast in preeclampsia which comes into maternal circulation and causes maternal inflammatory syndrome. ${ }^{[6]}$
However confusion exists regarding the type of trophoblast showing increase in apoptosis. Thus the aim of the study is to calculate apoptotic index in cytotrophoblast and syncytiotrophoblast in preeclampsia and IUGR and compare it with placenta from normal pregnancy

\section{Materials and Methods}

Study was carried out in the department of pathology in our college with case inputs from Department of Obstetrics and Gynecology from February 2016 to May 2016(4 months)

Sample size was estimated by using pilot study and Mean Apoptotic index number of cases. A total of fifty four Placentas were included in the study. 18/54 cases were from preeclampsia patients, $18 / 54$ cases were from IUGR patients, 18/54 were age matched controls

\section{Inclusion Criteria:}

Preeclampsia: Placenta of women with mild and severe preeclampsia without intrauterine growth retardation having blood pressure at or above $140 / 90 \mathrm{~mm}$ of $\mathrm{Hg}$ after 20 weeks of gestation with proteinuria

IUGR: Placenta from women having intrauterine growth retardation as diagnosed on weight for age in weeks by ultrasonography and Doppler studies. 
Controls: Gestational age matched placenta from pregnant women with normal blood pressure without proteinuria and without intrauterine growth retardation.

\section{Exclusion Criteria:}

Placenta from women having gestational diabetes mellitus, chronic hypertension, twin pregnancy, hypothyroid patient, congenital abnormality, associated medical condition like Heart disease, Systemic lupus erythematous, post term pregnancy, abruption placenta and asymmetrical IUGR were excluded from the study.

Method Of Collecting Data-The placenta was collected immediately after delivery from preeclampsia cases and control groups and was washed in running tap water to remove any blood clot. Gross examination was done noting weight, diameter, thickness, number of cotyledon on the maternal surface, calcification, infarction and umbilical cord vessels and knots. The placenta was cut in regular interval of $0.5 \mathrm{~cm}$ and gross lesions were identified (Bread slice technique). The whole specimen was left for fixation in $10 \%$ formalin for $24-48$ hours. Five sections were taken each from central and peripheral areas. Additional section was taken from grossly abnormal areas. Tissue was processed and paraffin blocks were made. Tissue sections of 4 micrometer thickness was cut from paraffin embedded blocks and stained by conventional Hematoxylin and eosin stain (H\&E)

Apoptotic cells were identified in the light microscopy by deep eosinophilia of cytoplasm, pyknotic round to crescentic irregular nucleus whereas apoptotic bodies/ nuclei appeared as tiny, round and pyknotic nuclear fragments. ${ }^{[7]}$ Apoptotic cells/bodies were counted at high field in the H\&E stained slides under oil immersion using Olympus microscope (100X) in both cytotrophoblasts (CT) and syncytiotrophoblasts (ST). Apoptotic index (AI) was calculated by counting total number of apoptotic bodies (AB) and dividing it by total number of nuclei counted multiplied by $100 .^{[8]}$ All the slides were reviewed by two authors .In case of any discrepancy it was discussed with the third author.
Statistical Analysis- Data was entered into Microsoft excel data sheet and was analyzed using SPSS 22 version software. Categorical data was represented in the form of Frequencies and proportions. Chi-square was used as test of significance. Continuous data was represented as mean and standard deviation. ANOVA (Analysis of Variance) was the test of significance to identify the mean difference between more than two groups. $\mathrm{p}$ value $<0.05$ was considered as statistically significant

\section{Result:}

Profile of subjects in the study are shown in table 1. No significant difference was seen in mean age between three groups. Mean baby weight was lower in IUGR and Preeclampsia than in Normal babies. This difference was statistically significant. Similarly statistically significance was seen in mean weight of placenta was significantly lower in IUGR and preeclampsia than in normal subjects. Location of Placenta and Membrane status between three groups are placed in table 2. There was no significant difference in location of placenta between three groups. Most common location in IUGR and Pre-eclampsia was Eccentric. In normal subjects most common placental location was central. Significant difference in membrane appearance was observed between three groups. In normal subjects majority of them had clear membrane, in preeclampsia and IUGR subject's majority had opaque membrane. Gross image of opaque preeclampsia placenta is shown in figure 1. Apoptotic bodies and Apoptotic index comparison between three groups are shown in table 3

Statistically significant difference was observed in $A B$ in $\mathrm{CT}, \mathrm{AB}$ in ST, AI in CT and AI in ST between three groups. All the parameters were lowest in Pre-eclampsia $<$ IUGR $<$ Normal. The apoptotic bodies in preeclampsia and IUGR placentas are shown in figure 2,3

\section{Discussion}

Pre-eclampsia causes considerable mortality and morbidity in both mother and fetus while IUGR is associated with poor pregnancy outcome. $\left.{ }^{[9}\right] \mathrm{A}$ variety of pathological stimuli is usually seen in both conditions. Apoptosis is a programmed cell death seen as a part of development, inflammation and malignancy. Like elsewhere apoptosis

Table 1: Profile of Subjects in the study.

\begin{tabular}{|l|c|c|c|c|c|c|c|}
\hline \multirow{2}{*}{} & \multicolumn{9}{|c|}{ Diagnosis } & \multirow{2}{*}{ P value } \\
\cline { 2 - 7 } & \multicolumn{2}{|c|}{ Normal } & \multicolumn{2}{c|}{ Pre-eclampsia } & \multicolumn{2}{c|}{ IUGR } & \\
\cline { 2 - 7 } & Mean & SD & Mean & SD & Mean & SD & \\
\hline Age of mother & 22.8 & 2.9 & 24.0 & 2.7 & 24.3 & 2.7 & 0.264 \\
\hline Baby weight & 3.2 & 0.5 & 2.6 & 0.4 & 2.2 & 0.1 & $<0.001^{*}$ \\
\hline Wt of Placenta & 433.6 & 78.8 & 361.1 & 80.6 & 272.8 & 38.0 & $<0.001^{*}$ \\
\hline
\end{tabular}


Table 2: Apoptotic bodies and Apoptotic index comparison between three groups

\begin{tabular}{|c|c|c|c|c|c|c|c|}
\hline & \multicolumn{6}{|c|}{ Diagnosis } & \multirow[t]{3}{*}{ P value } \\
\hline & \multicolumn{2}{|c|}{ Normal } & \multicolumn{2}{|c|}{ Pre-eclampsia } & \multicolumn{2}{|c|}{ IUGR } & \\
\hline & Mean & SD & Mean & SD & Mean & SD & \\
\hline$A B$ in $C T$ & 34.4 & 9.8 & 65.6 & 11.9 & 51.4 & 14.3 & $<0.001^{*}$ \\
\hline$A B$ in $S T$ & 40.5 & 8.8 & 82.9 & 15.9 & 74.2 & 11.1 & $<0.001^{*}$ \\
\hline AI IN CT & 6.8 & 2.0 & 13.1 & 2.4 & 10.3 & 2.9 & $<0.001^{*}$ \\
\hline $\mathrm{Al}$ in $\mathrm{ST}$ & 8.1 & 1.7 & 16.6 & 3.2 & 14.8 & 2.2 & $<0.001^{*}$ \\
\hline
\end{tabular}

Table 3: Location of Placenta and Membrane status between three groups.

\begin{tabular}{|c|c|c|c|c|c|c|c|c|}
\hline & & \multicolumn{6}{|c|}{ Diagnosis } & \multirow{3}{*}{$P$ value } \\
\hline & & \multicolumn{2}{|c|}{ Normal } & \multicolumn{2}{|c|}{ Preeclampsia } & \multicolumn{2}{|c|}{ IUGR } & \\
\hline & & Count & $\%$ & Count & $\%$ & Count & $\%$ & \\
\hline \multirow{3}{*}{$\begin{array}{l}\text { Location of } \\
\text { Placenta }\end{array}$} & Central & 8 & $44.4 \%$ & 4 & $22.2 \%$ & 6 & $33.3 \%$ & \multirow{3}{*}{0.406} \\
\hline & Eccentric & 6 & $33.3 \%$ & 12 & $66.7 \%$ & 9 & $50.0 \%$ & \\
\hline & Para central & 4 & $22.2 \%$ & 2 & $11.1 \%$ & 3 & $16.7 \%$ & \\
\hline \multirow{3}{*}{ Membrane } & Clear & 14 & $77.8 \%$ & 1 & $5.6 \%$ & 4 & $22.2 \%$ & \multirow{3}{*}{$<0.001^{*}$} \\
\hline & Opaque & 0 & $0.0 \%$ & 14 & $77.8 \%$ & 7 & $38.9 \%$ & \\
\hline & Semi opaque & 4 & $22.2 \%$ & 3 & $16.7 \%$ & 7 & $38.9 \%$ & \\
\hline
\end{tabular}

Table 4: Showing Comparison of different studies with the present study

\begin{tabular}{|c|c|c|c|c|c|c|}
\hline \multirow{2}{*}{ Study } & \multicolumn{2}{|c|}{ Normal } & \multicolumn{2}{|c|}{ IUGR } & \multicolumn{2}{|c|}{ Preeclampsia } \\
\hline & Synctio & Cyto & Synctio & Cyto & Synctio & Cyto \\
\hline Longtine MS etal ${ }^{11}$ (Caspase) & Low & Low & - & - & Lower & High \\
\hline Crocker IP etal ${ }^{12}$ (Annexin V) & Low & Low & High & High & Highest & Highest \\
\hline Present study(Light microscopy) & Low & Low & High & High & Highest & Highest \\
\hline
\end{tabular}

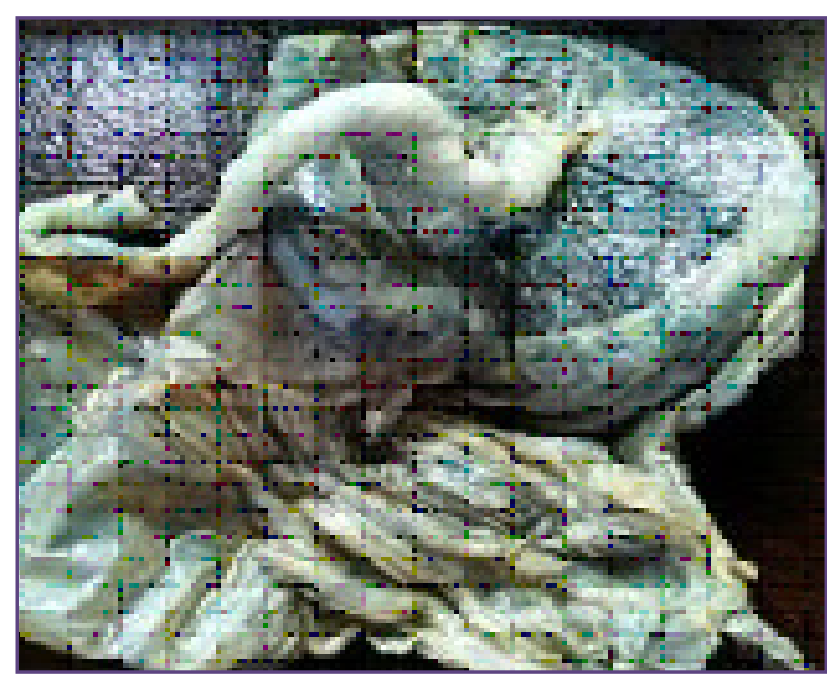

Fig. 1: Shows opaque membrane and centrally placed umbilical cord of placenta in pre-eclampsia.

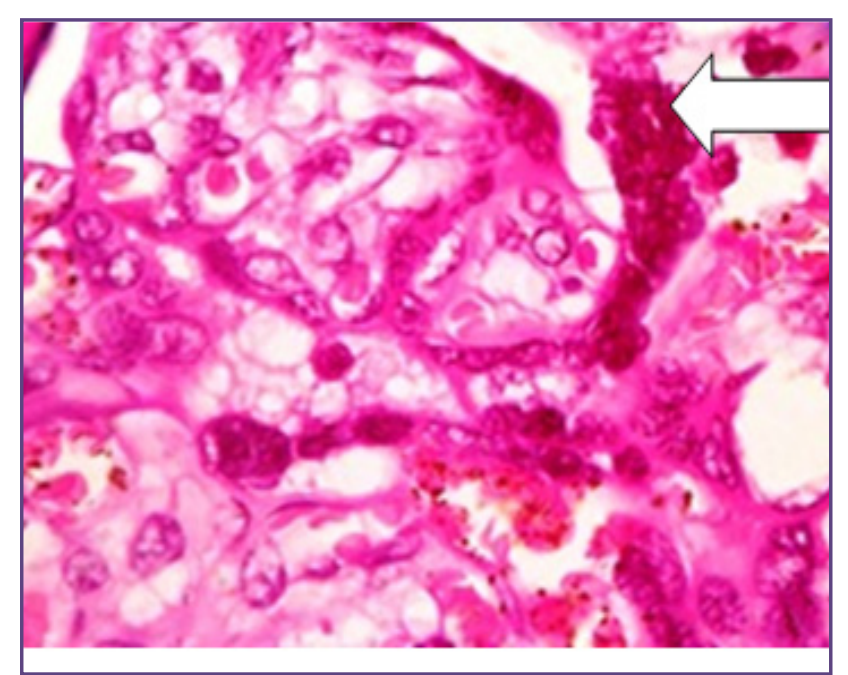

Fig. 2: Shows apoptotic nuclei in synctiocytotrophoblast in pre-eclampsia. $\mathrm{H} \& \mathrm{E}(100 \mathrm{X})$. 


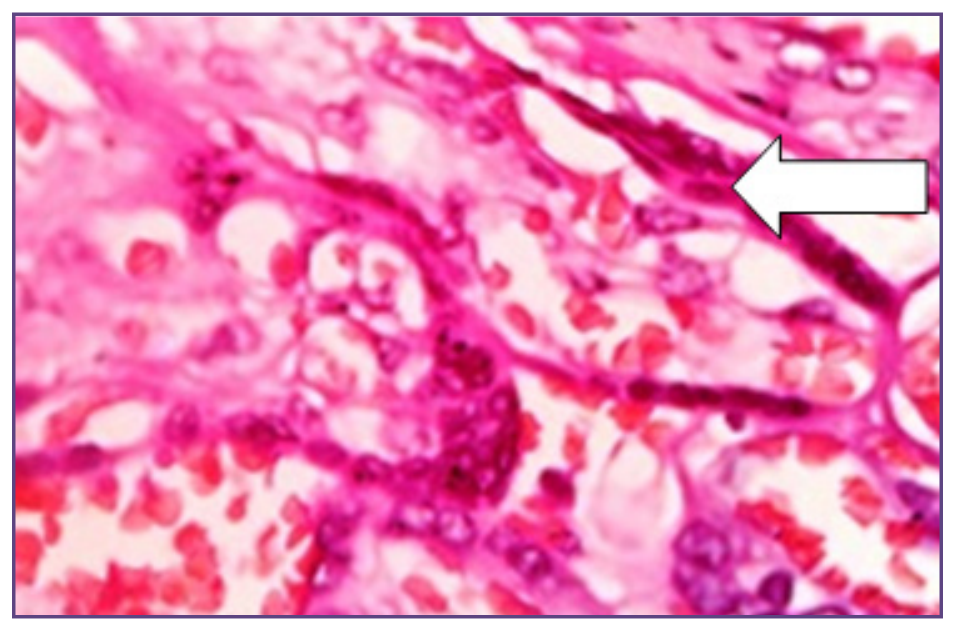

Fig. 3: Shows apoptotic nuclei in cytotrophoblast in IUGR placenta .H\&E (100X)

in placenta is a normal physiological process. The cytotrophoblast daughter cells differentiate and fuse to form the syncytiotrophoblast layer as normal turnover of trophoblast. Following differentiation and maturation within the syncytiotrophoblast layer the aging nuclei are shed by apoptosis and to a lesser extent, by necrosis, into the intervillous space where they are engulfed and cleared by macrophages. This phenomenon protects the mother from an imminent inflammatory response. This process is a continuous cycle that is carefully regulated throughout gestation. ${ }^{[10,11]}$ Many techniques are available to visualize apoptotic cell/nuclei. They can be best seen by using Electron microscopy which is a gold standard method, but cost and availability is a major limitation. Few studies are done using TUNEL method for staining apoptotic nuclei, however this technique detects necrotic cells in addition to apoptotic cell. ${ }^{[12]}$ Our study using light microscopy of placenta shows an incremental increase in apoptosis of cytotrophoblast, syncytiotrophoblast in placentas of normal, IUGR and preeclampsia pregnancies. In a study done by Longtine MS et al on pre eclamptic placentas, caspase mediated apoptosis was increased in cytotrophoblast as compared to syncytiotrophoblast. They said such a phenomenon acts as a protective mechanism in the placenta to prevent catastropic spreading of apoptosis. [13]

Another study done by Crocker et al concluded that increased apoptosis is seen in both cells of IUGR, preeclampsia cases. This trophoblastic apoptosis may be a reflection of unfavorable conditions in the placenta. ${ }^{[14]}$

In another study by Stephen C Smith, increased apoptosis was seen in syncytiotrophoblast, and stromal cells of IUGR placenta. They explained that this increase in apoptosis was a protective phenomenon to allow better transport of nutrients across the membrane thus resulting in smaller placentas and smaller babies. ${ }^{[15]}$

Few studies have shown apoptotic indices of $0.17 \%$, $0.24 \%, 0.39 \%$ was seen in normal, IUGR and preeclampsia placenta. This sequential increase was also seen in our study. ${ }^{[16,17]}$ Apoptotic cascade initiation phase takes place in cytotrophoblast while execution phase in Syncytiotrophoblasts. Apoptotic nuclei accumulates as syncytial knots. Though these particles are released into maternal circulation. An increase in the circulation of these particles may be a reason for maternal endothelial disruption in preeclampsia. This theory was supported by study by Goswami et al who detected apoptotic syncytiotrophoblastic microparticles (STMP) in the serum by Elisa method. They found maximum increase in these particles in patients with preeclampsia as compared to IUGR and normal placentas which may be the reason for development of maternal inflammatory syndrome in preeclampsia. ${ }^{[18]}$

Placenta in IUGR babies are known to have capillary abnormalities and villous atrophy which can be the cause of impaired oxygen supply to the placenta leading to increased apoptosis in placenta and small placentas . ${ }^{[19]}$

Major limitation of our study is the use of light microscopy for detecting apoptosis. However use of oil immersion and counting of many cells may help in overcoming this limitation to some extent.

\section{Conclusion}

Apoptosis is a feature of villous trophoblast in placenta and is significantly increased in pregnancy complicated by pre-eclampsia and IUGR. The Apoptotic index of cytotrophoblast and syncytiotrophoblast nuclei in preeclampsia and IUGR are significantly higher than 
in normal placenta. Further studied using markers for apoptotic nuclei are needed for better understanding of pathophysiology of these conditions.

\section{Reference}

1. Hutcheon JA, Lisonkova S, Joseph KS. Epidemiology of pre-eclampsia and the other hypertensive disorders of pregnancy. Best Pract Res Clin Obstet Gynecol 2011; 25:242-46.

2. Bahr BL, Price MD, Merrill D, Mejia C, Call L, Bears D, et al. Different expression of placental pyruvate kinase in normal, preeclamptic and intrauterine growth restriction pregnancies. Placenta 2014;35:883-90.

3. Sharp AN, Heazell EPA, Crocker IP, Mor G. Placental Apoptosis in Health and Disease. Am J Reprod Immunol 2010; 64:159-69.

4. Staff A, Dechend R, Pijnenborg R. Acute Atherosis and Vascular Remodeling in Preeclampsia-Novel Aspects for Atherosclerosis and Future Cardiovascular Health. Hypertension 2010; 56:1026-34.

5. Grill S, Rusterholz C, Zanetti DR, Tercanli S, Holzgreve W. Hanhn S etal. Potential markers of preeclampsia-a review. Reprod Biol Endocrinol 2009; 7:70-75.

6. Guller S. Role of the syncytium in placenta-mediated complications of pre-eclampsia. Thromb Res 2009; 124:389-92.

7. Jain A, Maheshwari V, Alam K, Mehdi G, Sharma SC. Apoptosis in premalignant and malignant squamous cell lesions of the oral cavity: A light microscopic study. IJPM 2009; 5 2:164-66

8. Potten CS. What is an apoptotic index measuring? A commentary. Brit J Cancer 1996; 74:1743-48

9. Huppertz B. Placental origins of preeclampsia :challenging and current hypothesis. Hypertension 2008;51:970-75.

10. Tannetta DS, Dragovic RA, Gardiner C, Redman CW, Sargent IL. Characterisation of syncytiotrophoblast vesicles in normal pregnancy and pre-eclampsia:Expression of Flt1and Endoglin. PLOS ONE 2013;8
11. Roberts JM, Escudero C. The placenta in preeclampsia. Hypertens Pregnancy 2012;2:72-83.

12. Florio P, Marinoni E, Dilorio R, Bashir M, Ciotti S, Sacchi R. Urinary S100B Protein Concentrations are Increased in Intrauterine Growth-Retarded Newborns. Pediatrics 2006; 118:747-54.

13. Longtine MS, Chen B, Odibo AO, Zhong Y, Nelson DM. Caspase mediated apoptosis of trophoblasts in term human placental villi is restricted to cytoptrophoblasts and absent from syncytiotrobhoblast. Reproduction. 2012;143:107-21.

14. Crocker IP, Cooper S, Ong SC, Baker PN. Differences in Apoptotic Susceptibility of Cytotrophoblasts and Syncytiotrophoblasts in Normal Pregnancy to Those Complicated with Preeclampsia and Intrauterine Growth Restriction. Am J Pathol 2003;162:637-43.

15. Smith SC, Baker PN, Symonds EM. Increased placental apoptosis in intrauterine growth restriction. Am J Obstet Gynecol 1997; 177:1395 - 1401

16. Chen B, Longtine MS, Sadovsky Y, Nelson DM. Hypoxia downregulates p53 but induces apoptosis and enhances expression of BAD in cultures of human syncytiotrophoblasts. Am J Physiol Cell Physiol. 2010;299:C968-C976.

17. Crocker IP, Barratt S, Kaur M, Baker PN. The invitro characterization of induced apoptosis in placental cytotrophoblasts and syncytiotrophoblasts. Placenta. 2001;22:822-830.

18. Goswami D, Tannetta DS, Magee LA, Fuchisawa A, Redman CWG, Sargent IL, von Dadelszen P. Excess syncytiotrophoblast microparticle shedding is a feature of early-onset pre-eclampsia, but not normotensive intrauterine growth restriction. Placenta 2006; 27: 56-61. doi.org/10.1016/j.

19. Krcbs C, Macara LM, Lciscf R, Bowman AW, Greet IA, Kingdom JC. Intrauterine growth restriction with absent enddiastolic flow velocity in the umbilical artery is associated with maldevelopment of the placenta! terminal villous tree. Am J Obstet Gynecol 1996;175:1534-42.

*Corresponding author:

Dr Hemalatha. A, Associate Professor, Department of Pathology, Sri Devaraj Urs Medical College, Tamaka, Kolar 563103,India

Phone: +91 9972212324

Email: drhemashashi@gmail.com

Financial or other Competing Interests: None. 